\title{
SPACE-LIKE SUBMANIFOLDS WITH PARALLEL MEAN CURVATURE IN DE SITTER SPACES
}

\author{
CHONGZHEN OUYANG and ZHENQI LI
}

(Received 10 December 1998; revised 28 June 1999)

\author{
Communicated by K. Ecker
}

\begin{abstract}
This paper investigates complete space-like submanifold with parallel mean curvature vector in the de Sitter space. Some pinching theorems on square of the norm of the second fundamental form are given.

2000 Mathematics subject classification: primary 53C40; secondary 53C42.

Keywords and phrases: de Sitter space, space-like submanifold, mean curvature vector, parallel.
\end{abstract}

\section{Introduction}

A de Sitter space $S_{p}^{n+p}(1)$ is an $(n+p)$-dimensional connected complete pseudoRiemannian manifold of index $p$ with constant curvature 1. Goddard [3] conjectured that complete space-like hypersurface in $S_{1}^{n+1}(1)$ with constant mean curvature $H$ must be totally umbilical. In 1987, Akutagawa [1] and Ramanathan [6] proved independently the conjecture is true if $H^{2} \leq 1$ when $n=2$ and $n^{2} H^{2}<4(n-1)$ when $n \geq 3$. This statement has been generalized by Cheng [2] to complete space-like submanifolds in $S_{p}^{n+p}(1)$ with parallel mean curvature vector. In [5], we proved that complete space-like hypersurface $M$ in $S_{1}^{n+1}(1)$ with constant mean curvature is totally umbilical if $S \leq 2 \sqrt{n-1}$, where $S$ is the square of the second fundamental form. Moreover, $S=2 \sqrt{n-1}$ only if $n=2$ and $M$ is flat.

In the present paper we shall prove the following

THEOREM 1. Let $M$ be a complete space-like n-dimensional submanifold in the de Sitter space $S_{p}^{n+p}(1)$ with parallel mean curvature vector $\eta$. Denote by $S$ the square of norm of second fundamental form.

Project supported by NSFC (Grant No. 19871038) and NSF of Jiangxi Province.

(C) 2000 Australian Mathematical Society 0263-6115/2000\$A2.00+0.00 
(i) If $S \leq\left((2 n \sqrt{n-1} /(n+2 \sqrt{n-1}))\left(1+\|\eta\|^{2}\right)\right.$, then $M$ is totally umbilical and lies in a totally geodesic submanifold $S_{1}^{n+1}(1)$ of $S_{p}^{n+p}(1)$. Moreover, $M$ is isometric to a sphere $S^{n}(\sqrt{n /(n-S)})$ of radius $\sqrt{n /(n-S)}$ or a plane $\mathbb{R}^{2}$ in case $S=n=2$.

(ii) If $S \leq\left((2 n \sqrt{n-1} /(n-2))\left(1-\|\eta\|^{2}\right)(n>2)\right.$, then $M$ lies in a totally geodesic submanifold $S_{1}^{n+1}(1)$ of $S_{p}^{n+p}(1)$.

\section{Preliminaries}

Let $M$ be an $n$-dimensional space-like submanifold of $S_{p}^{n+p}(1)$. Locally we choose a pseudo-Riemannian orthonormal frame $\left\{e_{1}, \ldots, e_{n+p}\right\}$ in $S_{p}^{n+p}(1)$ such that, restricted to $M, e_{1}, \ldots, e_{n}$ is tangent to $M$. Throughout this paper the following convention on the ranges of indices is used unless otherwise stated

$1 \leq A, B, C, D, \ldots \leq n+p, \quad 1 \leq i, j, k, l, \ldots \leq n, \quad n+1 \leq \alpha, \beta, \ldots \leq n+p$.

Let $\left\{\omega_{1}, \ldots, \omega_{n+p}\right\}$ be the dual coframe of $\left\{e_{A}\right\}$. The pseudo-Riemannian metric on $S_{p}^{n+p}(1)$ is

$$
d s^{2}=\sum_{A} \varepsilon_{A} \omega_{A}^{2}
$$

where $\varepsilon_{1}=\cdots=\varepsilon_{n}=1, \varepsilon_{n+1}=\cdots=\varepsilon_{n+p}=-1$. The structure equations are

$$
\begin{gathered}
d \omega_{A}=-\sum_{B} \omega_{A B} \wedge \omega_{B}, \quad \varepsilon_{A} \omega_{A B}+\varepsilon_{B} \omega_{B A}=0, \\
d \omega_{A B}=-\sum_{C} \omega_{A C} \wedge \omega_{C B}+\varepsilon_{B} \omega_{A} \wedge \omega_{B} .
\end{gathered}
$$

Restricted to $M$ we have

$$
\begin{gathered}
d s^{2}=\sum_{i}\left(\omega_{i}\right)^{2} \\
\omega_{\alpha i}=\sum_{j} h_{i j}^{\alpha} \omega_{j} \\
R_{i j k l}=\delta_{i k} \delta_{j l}-\delta_{i l} \delta_{j k}-\sum_{\alpha}\left(h_{i k}^{\alpha} h_{j l}^{\alpha}-h_{i l}^{\alpha} h_{j k}^{\alpha}\right) \\
R_{\alpha \beta j k}=-\sum_{i}\left(h_{i j}^{\alpha} h_{i k}^{\beta}-h_{i k}^{\alpha} h_{i j}^{\beta}\right)
\end{gathered}
$$

where $R_{i j k l}$ are the components of the curvature tensor of $M, R_{\alpha \beta j k}$ the components of the curvature tensor of the normal bundle $T^{\perp} M$, and $h_{i j}^{\alpha}$ the components of the second fundamental form $\sigma=\sum_{\alpha, i, j} h_{i j}^{\alpha} \omega_{i} \otimes \omega_{j} \otimes e_{\alpha}$. We define $h_{i j k}^{\alpha}$ by

$$
\sum_{k} h_{i j k}^{\alpha} \omega_{k}=d h_{i j}^{\alpha}-\sum_{k} h_{k j}^{\alpha} \omega_{k i}-\sum_{k} h_{i k}^{\alpha} \omega_{k j}+\sum_{\beta} h_{i j}^{\beta} \omega_{\alpha \beta} \text {. }
$$


Then we have the Codazzi equation

$$
h_{i j k}^{\alpha}=h_{i k j}^{\alpha} .
$$

The square $S$ of the norm of $\sigma$ is

$$
S=\|\sigma\|^{2}=\sum_{\alpha, i, j}\left(h_{i j}^{\alpha}\right)^{2}
$$

and the mean curvature vector $\eta$ of $M$ is given by

$$
\eta=\frac{1}{n} \operatorname{tr} \sigma=\frac{1}{n} \sum_{\alpha, i} h_{i i}^{\alpha} e_{\alpha} .
$$

We need the following lemmas.

LeMmA 1 ([7]). Let $A, B$ be symmetric $n \times n$ matrices satisfying $A B=B A$ and $\operatorname{tr} A=\operatorname{tr} B=0$. Then

$$
\left|\operatorname{tr} A^{2} B\right| \leq \frac{n-2}{\sqrt{n(n-1)}}\left(\operatorname{tr} A^{2}\right)\left(\operatorname{tr} B^{2}\right)^{1 / 2} .
$$

LEMMA $2([4,9])$. Let $M$ be a complete Riemannian manifold whose Ricci curvature is bounded from below. If $F$ is a $C^{2}$-function bounded from above on $M$, then for any $\varepsilon>0$, there is a point $x \in M$ such that

$$
\sup F-\varepsilon<F(x), \quad\|\nabla F\|(x)<\varepsilon, \quad \Delta F(x)<\varepsilon .
$$

\section{Proof of Theorem 1}

Set $\|\eta\|=\sqrt{|\langle\eta, \eta\rangle|}$. Since $\|\eta\|^{2} \leq S / n$ and the equality holds only on set of umbilical points, the condition $S \leq((2 n \sqrt{n-1}) /(n+2 \sqrt{n-1}))\left(1+\|\eta\|^{2}\right)$ implies that $S \leq 2 \sqrt{n-1}$ and the equality holds only on the set of umbilical points. Therefore the theorem follows from [5] if $p=1$ and [2] if $\eta=0$.

We now suppose that $p \geq 2$ and $\eta \neq 0$. Thus we can choose the frame $e_{1}, \ldots, e_{n+p}$ as in Section 2 with $e_{n+1}=\eta /\|\eta\|$. Then

$$
\|\eta\|=\frac{1}{n} \sum_{i} h_{i i}^{n+1}, \quad \sum_{i} h_{i i}^{\alpha}=0, \quad(\alpha>n+1) .
$$

Since $\eta$ is parallel in $T^{\perp} M$, we know $\|\eta\|$ is constant and $\omega_{\alpha n+1}=0$. Consequently, $R_{\alpha n+1 j k}=0$. From (2.7) we have

$$
\sum_{i} h_{i j}^{\alpha} h_{i k}^{n+1}=\sum_{i} h_{i k}^{\alpha} h_{i j}^{n+1}
$$


Denote by $H_{\alpha}$ the matrix $\left(h_{i j}^{\alpha}\right)$ for $\alpha=n+1, \ldots, n+p$. (3.2) means

$$
H_{\alpha} H_{n+1}=H_{n+1} H_{\alpha} .
$$

We define $h_{i j k l}^{\alpha}$ by

$$
\sum_{l} h_{i j k l}^{\alpha} \omega_{l}=d h_{i j k}^{\alpha}-\sum_{l}\left(h_{l j k}^{\alpha} \omega_{l i}+h_{i l k}^{\alpha} \omega_{l j}+h_{i j l}^{\alpha} \omega_{l k}\right)+\sum_{\beta} h_{i j k}^{\beta} \omega_{\alpha \beta} .
$$

The Laplacian of $h_{i j}^{\alpha}$ is defined by

$$
\Delta h_{i j}^{\alpha}=\sum_{k} h_{i j k k}^{\alpha} .
$$

From (2.9), (3.1), and (3.4) we obtain

$$
\Delta h_{i j}^{\alpha}=\sum_{k, l}\left(h_{k l}^{\alpha} R_{l i j k}+h_{i l}^{\alpha} R_{l k j k}\right)-\sum_{\beta, k} h_{k i}^{\beta} R_{\alpha \beta j k} .
$$

Then by (2.6), (2.7), and (3.6) we have

$$
\begin{aligned}
\sum_{i, j} h_{i j}^{n+1} \Delta h_{i j}^{n+1}= & n \operatorname{tr} H_{n+1}^{2}-n^{2}\|\eta\|^{2}-n\|\eta\| \operatorname{tr} H_{n+1}^{3}+\sum_{\beta}\left[\operatorname{tr}\left(H_{n+1} H_{\beta}\right)\right]^{2} \\
\sum_{i, j} h_{i j}^{\alpha} \Delta h_{i j}^{\alpha}= & n \operatorname{tr} H_{\alpha}^{2}-n\|\eta\| \operatorname{tr}\left(H_{\alpha}^{2} H_{n+1}\right)-\sum_{\beta} \operatorname{tr}\left(H_{\alpha} H_{\beta}-H_{\beta} H_{\alpha}\right)^{2} \\
& +\sum_{\beta}\left[\operatorname{tr}\left(H_{\alpha} H_{\beta}\right)\right]^{2}, \quad(\alpha>n+1) .
\end{aligned}
$$

Set $B=H_{n+1}-\|\eta\| I$. By means of (3.1) and (3.3) we get

$$
\operatorname{tr} B=0, \quad \operatorname{tr} H_{\alpha}=0, \quad H_{\alpha} B=B H_{\alpha}, \quad(\alpha>n+1) .
$$

By virtue of Lemma 1,

$$
\left|\operatorname{tr}\left(H_{\alpha}^{2} B\right)\right| \leq \frac{n-2}{\sqrt{n(n-1)}} \operatorname{tr} H_{\alpha}^{2} \sqrt{\operatorname{tr} B^{2}}, \quad(\alpha>n+1) .
$$

Since

$$
\begin{aligned}
\operatorname{tr}\left(H_{\alpha}^{2} B\right) & =\operatorname{tr}\left(H_{\alpha}^{2} H_{n+1}\right)-\|\eta\| \operatorname{tr} H_{\alpha}^{2}, \quad(\alpha>n+1) \\
\operatorname{tr} B^{2} & =\operatorname{tr} H_{n+1}^{2}-n\|\eta\|^{2},
\end{aligned}
$$

from (3.10) we get

$$
\operatorname{tr}\left(H_{\alpha}^{2} H_{n+1}\right) \leq\left[\|\eta\|+\frac{n-2}{\sqrt{n(n-1)}} \sqrt{\operatorname{tr} H_{n+1}^{2}-n\|\eta\|^{2}}\right] \operatorname{tr} H_{\alpha}^{2} .
$$


Taking $A=B$ in Lemma 1 we obtain

$$
\left|\operatorname{tr} B^{3}\right| \leq \frac{n-2}{\sqrt{n(n-1)}}\left(\sqrt{\operatorname{tr} B^{2}}\right)^{3}
$$

and therefore,

(3.14) $\quad \operatorname{tr} H_{n+1}^{3} \leq 3\|\eta\| \operatorname{tr} H_{n+1}^{2}-2 n\|\eta\|^{3}+\frac{n-2}{\sqrt{n(n-1)}}\left(\operatorname{tr} H_{n+1}^{2}-n\|\eta\|^{2}\right)^{\frac{3}{2}}$.

Let $T=\operatorname{tr} H_{n+1}^{2}$ and $U=\sum_{\alpha>n+1} \operatorname{tr} H_{\alpha}^{2}$. Then $S=T+U$ and

$$
\begin{aligned}
\frac{1}{2} \Delta T & =\sum_{i j k}\left(h_{i j k}^{n+1}\right)^{2}+\sum_{i j} h_{i j}^{n+1} \Delta h_{i j}^{n+1} \\
& \geq\left(T-n\|\eta\|^{2}\right)\left[n+T-2 n\|\eta\|^{2}-\frac{n-2}{\sqrt{n(n-1)}} n\|\eta\| \sqrt{T-n\|\eta\|^{2}}\right]
\end{aligned}
$$

(3.16) $\frac{1}{2} \Delta U \geq \sum_{\alpha>n+1} \sum_{i j} h_{i j}^{\alpha} \Delta h_{i j}^{\alpha}$

$$
\geq n U\left[1-\|\eta\|^{2}-\frac{n-2}{\sqrt{n(n-1)}}\|\eta\| \sqrt{T-n\|\eta\|^{2}}\right]
$$

where we have used (3.7), (3.8), (3.12), and (3.14).

Since

$$
\begin{aligned}
& -2(n-2) \sqrt{n}\|\eta\| \sqrt{T-n\|\eta\|^{2}} \\
& =\left[(\sqrt{n-1}+1) \sqrt{T-n\|\eta\|^{2}}-(\sqrt{n-1}-1) \sqrt{n}\|\eta\|\right]^{2} \\
& \quad+4 n \sqrt{n-1}\|\eta\|^{2}-(n+2 \sqrt{n-1}) T
\end{aligned}
$$

we have

$$
\begin{aligned}
& \frac{1}{2} \Delta T \geq n\left(T-n\|\eta\|^{2}\right)\left(1-\frac{1}{2 \sqrt{n-1}} T\right), \\
& \frac{1}{2} \Delta U \geq n U\left(1+\|\eta\|^{2}-\frac{n+2 \sqrt{n-1}}{2 n \sqrt{n-1}} T\right) .
\end{aligned}
$$

By means of (2.6), we have

$$
R_{i j}=(n-1) \delta_{i j}+\sum_{k, \alpha} h_{i k}^{\alpha} h_{j k}^{\alpha}-\sum_{\alpha} h_{i j}^{\alpha} \sum_{k} h_{k k}^{\alpha}
$$

where $R_{i j}$ are the components of the Ricci tensor of $M$. Thus

$$
R_{i i} \geq n-1+\left(h_{i i}^{n+1}\right)^{2} \geq(n-1)-\frac{n^{2}}{4}\|\eta\|^{2} .
$$


Taking $F=-(U+1)^{-1 / 2}$ in Lemma 2, we know for any $\varepsilon>0$ there is $x \in M$ such that

$$
\sup F-\varepsilon<F(x), \quad\|\nabla F\|(x)<\varepsilon, \quad \Delta F(x)<\varepsilon .
$$

Since $\Delta F=-\frac{1}{2} F^{3} \Delta U+3 F^{-1}\|\nabla F\|^{2}$, we have

$$
\frac{1}{2} F^{4}(x) \Delta U(x)=3\|\nabla F\|^{2}(x)-F(x) \Delta F(x)<3 \varepsilon^{2}-\varepsilon F(x) .
$$

Thus, for any convergent sequence $\left\{\varepsilon_{m}\right\}$ with $\varepsilon_{m}>0$ and $\lim _{m \rightarrow \infty} \varepsilon_{m}=0$, there is a point sequence $\left\{x_{m}\right\}$ such that $\left\{F\left(x_{m}\right)\right\}$ satisfies (3.20) and $\lim _{m \rightarrow \infty} F\left(x_{m}\right)=F_{0}=$ $\sup F$, and therefore $\lim _{m \rightarrow \infty} U\left(x_{m}\right)=U_{0}=\sup U$.

On the other hand, from (3.21) we have

$$
\frac{1}{2} F^{4}\left(x_{m}\right) \Delta U\left(x_{m}\right)<3 \varepsilon_{m}^{2}-\varepsilon_{m} F\left(x_{m}\right)
$$

and the right hand side converges to 0 because $-1 \leq F \leq 0$. Accordingly for any $\varepsilon \in(0,2)$, there is $m_{\varepsilon}$ such that for $m>m_{\varepsilon}$,

$$
F^{4}\left(x_{m}\right) \Delta U\left(x_{m}\right) \gtrless \varepsilon .
$$

(3.18) and (3.22) yield

$$
\varepsilon\left[U\left(x_{m}\right)+1\right]^{2}>2 n U\left(x_{m}\right)\left[1+\|\eta\|^{2}-\frac{n+2 \sqrt{n-1}}{2 n \sqrt{n-1}} T\left(x_{m}\right)\right] .
$$

Under the hypothesis of (i) in Theorem 1, we have $\left(1+\|\eta\|^{2}\right) \geq((n+2 \sqrt{n-1}))$ $(2 n \sqrt{n-1}))(T+U)$. Hence, from (3.23) we get

$$
\varepsilon\left[U\left(x_{m}\right)+1\right]^{2}>\left(\frac{n}{\sqrt{n-1}}+2\right)\left[U\left(x_{m}\right)\right]^{2},
$$

which implies $\left\{U\left(x_{m}\right)\right\}$ is bounded and $U_{0}=0$. Thus $U=0$. Using the method of Yau [8] we know $M$ lies in a totally geodesic submanifold $S_{1}^{n+1}(1)$ of $S_{p}^{n+p}(1)$. Since $U=0$, we know $S=T$. The inequality (3.17) becomes

$$
\frac{1}{2} \Delta S \geq n\left(S-n\|\eta\|^{2}\right)\left(1-\frac{1}{2 \sqrt{n-1}} S\right) .
$$

Since $S \geq n\|\eta\|^{2}$ and $S \leq((2 n \sqrt{n-1}) /(n+2 \sqrt{n-1}))\left(1+\|\eta\|^{2}\right) \leq 2 \sqrt{n-1}$, (3.24) shows $\Delta S \geq 0$. Taking $F=-\left(S-n\|\eta\|^{2}+1\right)^{-1 / 2}$, in the same way as above we can prove $S-n\|\eta\|^{2}=0$. (Noting that $S=2 \sqrt{n-1}$ implies $S=n\|\eta\|^{2}$.) 
So $M$ is totally umbilical. From (2.6) we know $M$ has constant sectional curvature $K=1-S / n$. If $n \geq 3$, then $S<n$ by $S \geq 2 \sqrt{n-1}$, and $K>0, M$ is isometric to the sphere $S^{n}(r)$ of radius $r=\sqrt{n /(n-S)}$. If $n=2$, then either $M$ is flat (when $S=2$ ) or is isometric to $S^{2}(\sqrt{2 /(2-S)})$ (when $S<2$ ).

Under the hypothesis of (ii) in Theorem 1 we have

$$
(T+U) \leq \frac{2 n \sqrt{n-1}}{n-2}\left(1-\|\eta\|^{2}\right) .
$$

Noting $2 \sqrt{n}\|\eta\| \sqrt{T-n\|\eta\|^{2}} \leq T$, from (3.16) we have

$$
\frac{1}{2} \Delta U \geq n U\left[1-\|\eta\|^{2}-\frac{n-2}{2 n \sqrt{n-1}} T\right] \geq \frac{n-2}{2 \sqrt{n-1}} U^{2} \geq 0 .
$$

Applying Lemma 2 to $U$ we can get $U=0$ and therefore $H_{\alpha}=0$ for all $\alpha>n+1$. Using the method of Yau [8] we know $M$ lies in a totally geodesic submanifold $S_{1}^{n+1}(1)$ of $S_{p}^{n+p}(1)$. We then complete the proof of Theorem 1 .

\section{References}

[1] K. Akutagawa, 'On space-like hypersurfaces with constant mean curvature in the de Sitter space', Math. Z. 196 (1987), 13-19.

[2] Q. Cheng, 'Complete space-like submanifolds in a de Sitter space with parallel mean curvature vector', Math. Z. 206 (1991), 333-339.

[3] A. J. Goddard, 'Some remarks on the existence of spacelike hypersurfaces of constant mean curvature', Math. Proc. Cambridge Philos. Soc. 82 (1977), 489-495.

[4] H. Omori, 'Isometric immersions of Riemannian manifolds', J. Math. Soc. Japan 19 (1967), 205214.

[5] C. Ouyang and Z. Li, 'Complete space-like hypersurfaces with constant mean curvature in the de Sitter spaces', Chinese Quart. J. Math., to appear.

[6] J. Ramanathan, 'Complete spacelike hypersurfaces of constant mean curvature in a de Sitter space', Indiana Univ. Math. J. 36 (1987), 349-359.

[7] W. Santos, 'Submanifolds with parallel mean curvature in spheres', Tôhoku Math. J. 46 (1994), 405-415.

[8] S. T. Yau, 'Submanifolds with constant mean curvature I', Amer. J. Math. 96 (1974), 346-366.

[9] —_, 'Harmonic functions on complete Riemannian manifolds', Comm. Pure Appl. Math. 28 (1975), 201-228.

Department of Mathematics

Nanchang University

Nanchang 330047

P. R. of China

e-mail: zhengqili@263.net 